\title{
Trial Sets Name
}

National Cancer Institute

\section{Source}

National Cancer Institute. Trial Sets Name. NCI Thesaurus. Code C123981.

A character or string that represents the long name of the trial set. 